\title{
Flexible Switch Strategy for the Limited-Cycle with Multiple Periods
}

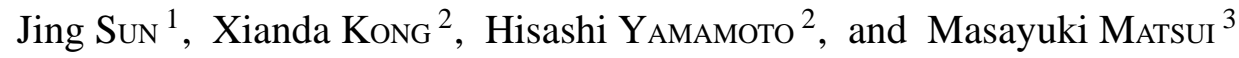 \\ 1 Graduate School of Engineering, Nagoya Institute of Technology, Gokiso-cho, Showa-ku, Nagoya, Aichi, 466-8555, Japan \\ 2 Department of Management Systems Engineering, Tokyo Metropolitan University, 6-6 Asahigaoka, Hino, Tokyo, 191-0065, Japan \\ 3 Department of IE and Management, Kanagawa University, 3-27-1 Rokkakubashi, Kanagawa-ku, Yokohama 221-8686, Japan
}

\begin{abstract}
This paper aims to derive optimal switch polity for the limited-cycle with multiple-periods. In a multi-period system (e.g., a production line) where target processing time exists, idle and delay risks occur repeatedly for multiple periods. Generally, whether this restriction is satisfied is not only dependent on the risks of this period but also the risks generated beforehand. We consider controlling the production process by switching the processing rate to a faster one at a given point (period) to minimize the total expected cost of the production process. In this paper, first, in order to consider the more flexible switch strategy, the optimal switching problem is discussed by subdividing the process with phases. Next, the mathematic formulations of switching problem based on the phase are analyzed. Finally, the policy of optimal switching strategy is investigated by Erlang numerical experiments.
\end{abstract}

Key Words : Limited-Cycle Problem, Multi-Period, Optimal Switching,Productione System, Manufacturing Line.

\section{Introduction}

Productivity improvement is not only one of the most important issues in manufacture management and operation research but also a significant factor affecting supply chain (SC). Many studies have analyzed different purposes for improving the system efficiency, such as assembly line balancing, inventory management, systems scheduling, optimal assignment and so on. As the most recent reports on the topices, Eswaramoorthi et al. presented an effective approach for balancing assembly operations with criteria to meet takt time by introducing a new parameter referred to as flow index [1]; Chen et al. presented optimal inventory control policy for periodic-review inventory systems with inventory-level-dependent demand [2]; Mejia et al. proposed the GAPN (genetic algorithms and Petri nets) approach, which combines the modelling power of petri nets with the optimisation capability of genetic algorithms (GAs) for manufacturing systems scheduling [3]; Hashimoto et al. cosidered the optimal arrangement to minimize the tatal expected risk on limited-cycle scheduling problem with multiple periods [4].

In any social system or production process with multi periods and predetermined target time, system idleness and process delay risks exists throughout the multiple periods. In such situations, delay of one process sometimes affect the delivery date of the entire process. This kind of problem is called "a limitedcycle problem with multiple periods" [5], and is seen in production lines, time-bucket balancing, production seat systems and so on [6]. In this paper, we discuss minimum expected risk (cost) in a production process, where the risk depends on the previous situation and occurs repeatedly throughout multiple

Corresponding Author: Jing Sun

Graduate School of Engineering, Nagoya Institute of Technology, Gokiso-cho, Showa-ku, Nagoya, Aichi, 466-8555, Japan sun.jing@nitech.ac.jp

(Received July 28, 2013)

(Revised December 10, 2013)

(Accepted December 23, 2013) periods

As limited-cycle problems of production line, Verzijl (1976) analyzed the element and construction of the production system [7]. Enns (2001) presented a framework for the analysis of delays within the production system [8]. Benders (2002) gave a review for the origin and solution of period batch control system [9]. Xia and Wu (2005) presented an easily implemented hybrid algorithm for the multi-objective flexible job-shop problem [10]. Recently, Wu and Zhou (2008) concerned with the problem in scheduling a set of jobs associated with random leadtime on a single machine so as to minimize the expected maximum lateness in stochastic environment [11].

As limited-cycle problems of operation management, Safaei and Tavakkoli-Moghaddam proposed an integrated mathematical model of the multi-period cell formation and production planning in a dynamic cellular manufacturing system to minimize costs through a mixed integer programming technique [12]. Porkka et al. proposed a mixed integer linear programming based capacitated lot sizing models that included carryovers incorporating set-up times with associated costs [13]. Moreno et al. El Hafsi and Bai employed an optimal multiperiod production plan for a single product over a finite planning horizon to minimize the total inventory and backlog costs by solving a nonlinear programming problem [14]. Li et al. described an optimal solution structure by the dynamic programming approach for a joint manufacturing and remanufacturing system in a multi-period horizon [15].

Under uncertain conditions, the result and efficiency of a certain production cycle period and a certain process are influenced not only by the risks that exist in the current period but also by the risks that existed in the foregoing periods. Therefore, we discuss the minimum expected risk of the case mentioned above, in which the risk depends on the foregoing situation and occurs repeatedly for multiple periods. Whether the process (period or site) satisfies the time limit (restriction) usually depends on the state of the foregoing process, as seen 


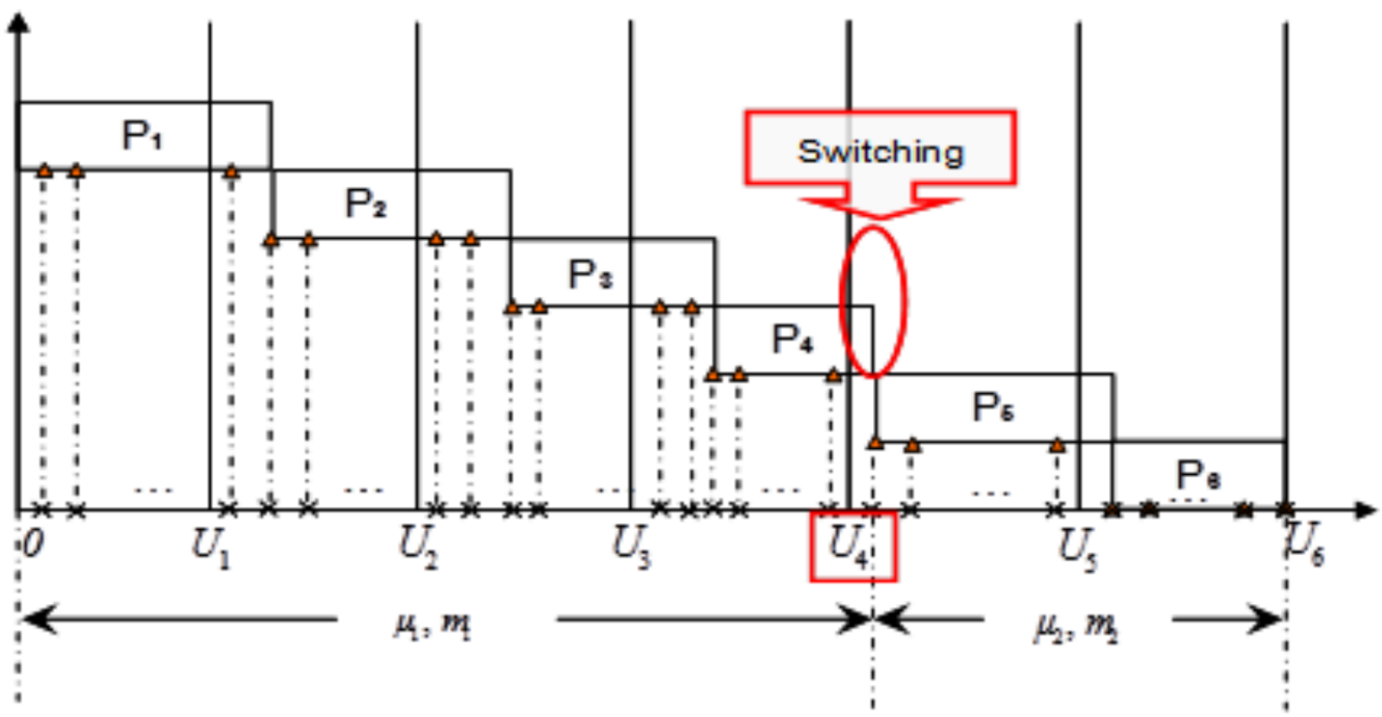

Fig. 1 Optimal Switching Problem ( $\mathrm{n}=6)$

in [7],[9],[16]. Which assignment of machines, workers, or jobs is the most efficient and economical (optimal assignment) is an important problem in load or risk planning (e.g., see [17],[18]), particularly in the case of risk that depends on the circumstances of a foregoing process (e.g., the case of a manufacturing line during a multi-period).

In our previous studies, Yamamoto et al. presented a recursive formula for the total expected risk and developed an algorithm for optimal assignments were for limited-cycle models with multiple periods based on the branch-and-bound method [1],[19]. Sun et al. proposed an optimal switching frequency model for short leadtime process [20]. Yamamoto et al.[21] and Kong et al.[22] described properties for an optimal assignment of two kinds of workers, among which one special worker exists.

In this paper, first, in order to consider the more flexible switch strategy, the optimal switching problem is discussed by subdividing the process with phases. Next, the mathematic formulations of switching problem based on the phase are analyzed. Finally, the policy of optimal switching strategy is investigated by Erlang numerical experiments.

\section{The Limited-Cycle Problem with Multiple Periods}

Before describing a limited-cycle problem with multiple periods, we consider a single limited-cycle problem. If the production time of one period is $T$ and the due time is $Z$, then the risks due to the length of the production time would occur, which are the risks by $T \leq Z$ and $T>Z$. In such situations, it can be noted that there is a trade-off problem in the two risks. This problem is shown as

$$
\min _{z}\left\{\beta_{1}(Z) P_{r}(T \leq Z)+\beta_{2}(Z) P_{r}(T>Z)\right\}
$$

where $\beta_{1}(Z)$ is the risk by $T \leq Z$, and $\beta_{2}(Z)$ is the risk by $T>Z$. This kind of problem is known as various problems of the reliability field, the problem of due time restriction and the newsboy problem (a well-known stock problem) [23]. A detailed model of a single limited-cycle can be found in reference [24].

This paper considers cases in which the above two risks not only occur in the single period, but also in multiple periods repeatedly. The problem of minimizing the expected risk in such a situation is a limited-cycle problem with multiple periods. The multi period problem could be classified according to whether the periods are independent or not.

For this problem, one result is the general form of production rate and waiting time by a station-centered approach as discussed in Matsui el al.[25]. The explicit form is obvious and consists of the product form in the period-independent case, such as a single line, but it is untraceable in the perioddependent case such as a mixed or tandem line. The mixed line has a absorbing barrier, but the tandem line has a reflective barrier at the end. This paper presents a cost approach for the latter.

\section{Optimal Switching Strategy}

In the production process of multi periods, delay of one process may influence the delivery date of an entire process. We consider controlling the production process by switching the processing rate to a faster one at a given point (time or period). The optimal switching problem is when the processing rate should be switched to minimize the total expectation cost. In this paper, we consider the optimal switching time to minimize the total expected cost of the production process. In our considered model shown in Figure 1, when the switching time is $k T, 1)$ if process $\mathrm{k}$ is complete at time $k T$, the processing rate is not changed and 2) if process $\mathrm{k}$ is not yet finished at $k T$, the pro- 
cessing rates is changed at the next prodess. Figure 1 shows an example of a switching time model in multiple periods, where period number $n$ is 6 and switching time $(k T)$ is $U_{4}=4 T$.

\subsection{The Assumption and Notation}

The Optimal Switching model for the multi limited-cycle is considered based on the following assumptions:

(1) One product is made by a process with n processes.

(2) For $i=1,2, \cdots, n$, the production time of process $\mathrm{i}$ is denoted by $T_{1}$ which is assumed to be Erlang distributed and statistically independent, respectively.

Where, $m_{i}$ is phases number of production process, $\mu_{1} / m_{1}$ is the usual processing rate, and $\mu_{2} / m_{2}$ is the emergency processing rate. In this paper, $\mu_{1} / m_{1}<\mu_{2} / m_{2}$ is assumed.

(3) For $i=1,2, \cdots, n$, the target production time of process $i$ is denoted by $U_{i}$, and the due time of the entire process (n periods) is $U_{n}$.

(4) $k T$ is switching time.

(5) The cost per unit time $\left(C_{s}^{(1)}\right)$ occurs when a process is executed before the target production time of the process.

(6) The cost per unit time $\left(C_{p}-(1)\right)$ occurs when a process is executed after the target production time of the process.

(7) When $X_{i}=\sum_{i=1}^{i} T_{i}>$ due time $\left(U_{n}\right)$ of n periods, the delay $\operatorname{cost} C_{p}$ occurs.

(8) When $X_{i}=\sum_{i=1}^{i} T_{i} \leq$ due time $\left(U_{n}\right)$ of $\mathrm{n}$ periods, the idle cost $C_{s}$ occurs.

Some notations are also defined.

For $i=1,2, \cdots, n$,

$C\left(T_{1}, T_{2}, \cdots, T_{n}\right):$ the total cost of the production process.

$C(i)$ : the production cost of period $i$.

$T_{i}$ : the production time of period $i$.

$X_{i}$ : the production time of i periods $\left(X_{i}=\sum_{i=1}^{i} T_{i}\right)$.

$\operatorname{Pr}\left(X_{n}>U_{n}\right)$ : the probability of delay.

$\operatorname{Pr}\left(X_{n} \leq U_{n}\right)$ : the probability of idle.

\subsection{Problem Formulation and Mathematical Analysis}

From assumptions (1)-(8) mentioned in Section 3.1, the object function is set as follows:

$$
\begin{gathered}
E\left[C\left(T_{1}, T_{2}, \ldots, T_{n}\right)\right]=\sum_{i=1}^{n} E[C(i)]+C_{p} \operatorname{Pr}\left(X_{n}>U_{n}\right) \\
+C_{s} \operatorname{Pr}\left(X_{n} \leq U_{n}\right)
\end{gathered}
$$

where, $E[C(i)]$ is the expected cost of period $i, C_{p} P_{r}\left(X_{n}>n T\right)$ is the delayed expected cost, $C_{S} P_{r}\left(X_{n} \leq n T\right)$ is the idle expected cost, and

$$
\begin{aligned}
& \text { For } i=1,2, \cdots, k, \\
& \qquad \begin{array}{l}
E[C(i)]= \\
E\left[\left(C_{p}^{(1)}-C_{s}^{(1)}\right) S_{i}+C_{s}^{(1)} T_{i}\right]
\end{array}
\end{aligned}
$$

$$
\begin{aligned}
& =\left(C_{p}^{(1)}-C_{s}^{(1)}\right) E\left[S_{i}\right]+C_{s}^{(1)} E\left[T_{i}\right] \\
& =\left(C_{p}^{(1)}-C_{s}^{(1)}\right) E\left[S_{i}\right]+\frac{m_{1} C_{s}^{(1)}}{\mu_{1}}
\end{aligned}
$$

for $i=k+1, k+2, \cdots, n$,

\section{$E[C(i)]$}

$$
\begin{aligned}
& =\left(C_{p}^{(1)}-C_{s}^{(1)}\right) E\left[S_{i} \mid \sum_{i=1}^{k} T_{i} \leq U_{k}\right] \operatorname{Pr}\left\{\sum_{i=1}^{k} T_{i} \leq U_{k}\right\} \\
& +\left(C_{p}^{(2)}-C_{s}^{(2)}\right) E\left[S_{i} \mid \sum_{i=1}^{k} T_{i}>U_{k}\right] \operatorname{Pr}\left\{\sum_{i=1}^{k} T_{i}>U_{k}\right\} \\
& +\frac{m_{1} C_{s}^{(1)}}{\mu_{1}} \operatorname{Pr}\left\{\sum_{i=1}^{k} T_{i} \leq U_{k}\right\}+\frac{m_{2} C_{s}^{(2)}}{\mu_{2}} \operatorname{Pr}\left\{\sum_{i=1}^{k} T_{i}>U_{k}\right\}
\end{aligned}
$$

Based on the relation between exponential distribution and Erlang distribution, the terms in equation (1) are given by following three theorems.

\section{Theorem 1.}

For $i=1,2, \cdots, n$, the expected cost of $n$ periods can be obtaind by Eq. (4):

$$
\begin{aligned}
& \sum_{i=1}^{n} E[C(i)] \\
& =\sum_{i=1}^{k} E[C(i)]+\sum_{i=k+1}^{n} E[C(i)] \\
& =\frac{m_{1} k}{\mu_{1}} C_{s}^{(1)}+\frac{m_{1}(n-k) C_{s}^{(1)}}{\mu_{1}}\left(1-\sum_{o=0}^{m_{1}(k-1)} \frac{\left(\mu_{1} U_{k}\right)^{o}}{o !} e^{-\mu_{1} U_{k}}\right) \\
& +\frac{m_{2}(n-k) C_{s}^{(2)}}{\mu_{2}} \sum_{o=0}^{m_{1}(k-1)} \frac{\left(\mu_{1} U_{k}\right)^{o}}{o !} e^{-\mu_{1} U_{k}} \\
& +\frac{m_{1}\left(C_{p}^{(1)}-C_{s}^{(1)}\right)}{\mu_{1}}\left(\sum_{i=1}^{k} \sum_{o=0}^{m_{1}(i-1)-1} \frac{\left(\mu_{1} U_{i}\right)^{o}}{o !} e^{-\mu_{1} U_{i}}\right. \\
& \left.+\sum_{i=k+1}^{n} \sum_{o=m_{1} k}^{m_{1}(i-2)+m_{1}-1} \sum_{o_{1}=m_{1} k}^{o} \frac{\left(\mu_{1} U_{k}\right)^{o_{1}}}{o_{1} !} \frac{\left\{\mu_{1}\left(U_{i}-U_{k}\right)\right\}^{o-o_{1}}}{\left(o-o_{1}\right) !} e^{-\mu_{1} U_{i}}\right) \\
& +\sum_{i=1}^{k} \sum_{o=m_{1}(i-1)}^{m_{1}(i-1)+m_{1}-1} \frac{\left(m_{1} i-o\right)\left(C_{p}^{(1)}-C_{s}^{(1)}\right)}{\mu_{1}} \frac{\left(\mu_{1} U_{i}\right)^{o}}{o !} e^{-\mu_{1} U_{i}} \\
& +\sum_{i=k+1}^{n} \sum_{o=m_{1} k}^{m_{1}(i-1)+m_{1}-1} \sum_{o_{1}=m_{1} k}^{o} \frac{\left(\mu_{1} U_{k}\right)^{o_{1}}}{o_{1} !} \frac{\left\{\mu_{1}\left(U_{i}-U_{k}\right)\right\}^{o-o_{1}}}{\left(o-o_{1}\right) !} e^{-\mu_{1} U_{i}} \\
& \frac{\left(m_{1} i-o\right)\left(C_{p}^{(1)}-C_{s}^{(1)}\right)}{\mu_{1}} \\
& +\frac{m_{2}\left(C_{p}^{(2)}-C_{s}^{(2)}\right)}{\mu_{2}}\left(\sum_{i=k+1}^{n} \sum_{o=0}^{m_{1} k-1} \frac{\left(\mu_{1} U_{i}\right)^{o}}{o !} e^{-\mu_{1} U_{i}}\right. \\
& +\sum_{i=k+1}^{n} \sum_{o=m_{1} k}^{m_{2}(i-2)+m_{2}-1-m_{2} k+m_{1} k} \int_{U_{k}}^{U_{i}} \frac{\left(\mu_{1} x\right)^{m_{1}(k-1)+m_{1}-1}}{\left\{m_{1}(k-1)+m_{1}-1\right\} !} e^{-\mu_{1} x}
\end{aligned}
$$




$$
\begin{aligned}
& \left.\frac{\left\{\mu_{2}\left(U_{i}-x\right)\right\}^{o-m_{2} k}}{\left(o-m_{2} k\right) !} e^{-\mu_{2}\left(U_{i}-x\right)} \mu_{1} d x\right) \\
& +\sum_{i=k+1}^{n} \sum_{o=m_{2}(i-1)-m_{2} k+m_{1} k}^{m_{2}(i-1)+m_{2}-1-m_{2} k+m_{1} k} \int_{U_{k}}^{U_{i}} \frac{\left(\mu_{1} x\right)^{m_{1}(k-1)+m_{1}-1}}{\left\{m_{1}(k-1)+m_{1}-1\right\} !} e^{-\mu_{1} x} \\
& \frac{\left\{\mu_{2}\left(U_{i}-x\right)\right\}^{o-m_{2} k}}{\left(o-m_{2} k\right) !} e^{-\mu_{2}\left(U_{i}-x\right)} \mu_{1} d x \\
& \frac{\left(m_{2} i-m_{2} k+m_{1} k-o\right)\left(C_{p}^{(2)}-C_{s}^{(2)}\right)}{\mu_{2}}
\end{aligned}
$$

\section{Theorem 2.}

For $l=1,2, \cdots, n$, the probability of delay can be obtaind by Eq. (5):

$$
\begin{aligned}
& \operatorname{Pr}\left\{\sum_{l=1}^{n} T_{l}>U_{n}\right\} \\
& =\sum_{o_{1}=m_{1} k}^{m_{1} n-1} \sum_{o_{2}=0}^{m_{1} n-1-o_{1}} \frac{\left(\mu_{1} U_{k}\right)^{o_{1}}}{o_{1} !} \frac{\left\{\mu_{1}\left(U_{n}-U_{k}\right)\right\}^{o_{2}}}{o_{2} !} e^{-\mu_{1} U_{n}} \\
& +\int_{U_{k}}^{U_{n}} \frac{\left(\mu_{1} x_{k}\right)^{m_{1} k-1}}{\left(m_{1} k-1\right) !} e^{-\mu_{1} x_{k}} \sum_{o=0}^{m_{2}(n-1)-m_{2} k} \frac{\left\{\mu_{2}\left(U_{n}-x_{k}\right)\right\}^{o}}{o !} \\
& e^{-\mu_{2}\left(U_{n}-x_{k}\right)} \mu_{1} d x_{k}+\sum_{o=0}^{m_{1} k-1} \frac{\left(\mu_{1} U_{n}\right)^{o}}{o !} e^{-\mu_{1} U_{n}}
\end{aligned}
$$

\section{Theorem 3.}

For $l=1,2, \cdots, n$, the probability of idle can be obtaind by Eq. (6):

$$
\begin{aligned}
& \operatorname{Pr}\left\{\sum_{l=1}^{n} T_{l} \leq U_{n}\right\} \\
& =\sum_{o_{1}=m_{1} k}^{\infty} \sum_{o_{2}=m_{1} n-o_{1}}^{\infty} \frac{\left(\mu_{1} U_{k}\right)^{o_{1}}}{o_{1} !} \frac{\left\{\mu_{1}\left(U_{n}-U_{k}\right)\right\}^{o_{2}}}{o_{2} !} e^{-\mu_{1} U_{n}} \\
& +\int_{U_{k}}^{U_{n}} \frac{\left(\mu_{1} x_{k}\right)^{m_{1} k-1}}{\left(m_{1} k-1\right) !} e^{-\mu_{1} x_{k}} \\
& \sum_{o=m_{2} n-m_{2} k}^{\infty} \frac{\left\{\mu_{2}\left(U_{n}-x_{k}\right)\right\}^{o}}{o !} e^{\mu_{2}\left(U_{n}-x_{k}\right)} \mu_{1} d x_{k}
\end{aligned}
$$

Also, the terms in equations (2) and (3) are given by following three Lemmas.

\section{Lemma 1.}

For $i=1,2, \cdots, k$,

$$
\begin{aligned}
& E\left[S_{i}\right]=\frac{m_{1}}{\mu_{1}} \sum_{o=0}^{m_{1}(i-1)-1} \frac{\left(\mu_{1} U_{i}\right)^{o}}{o !} e^{-\mu_{1} U_{i}} \\
& +\sum_{o=m_{1}(i-1)}^{m_{1}(i-1)+m_{1}-1} \frac{m_{1} i-o}{\mu_{1}} \frac{\left(\mu_{1} U_{i}\right)^{o}}{o !} e^{-\mu_{1} U_{i}}
\end{aligned}
$$

\section{Lemma 2.}

For $i=k+1, k+2, \cdots, n$,

$$
E\left[S_{i} \mid \sum_{i=1}^{k} T_{i} \leq U_{k}\right]
$$

$$
\begin{aligned}
& =\frac{m_{1}}{\mu_{1}} \frac{\sum_{o=m_{1} k}^{m_{1}(i-2)+m_{1}-1} \sum_{o_{1}=m_{1} k}^{o} \frac{\left(\mu_{1} U_{k}\right)^{o_{1}}}{o_{1} !} \frac{\left\{\mu_{1}\left(U_{i}-U_{k}\right)\right\}^{o-o_{1}}}{\left(o-o_{1}\right) !} e^{-\mu_{1} U_{i}}}{\sum_{o=m_{1} k}^{\infty} \frac{\left(\mu_{1} U_{k}\right)^{o}}{o !} e^{-\mu_{1} U_{k}}} \\
& +\frac{\sum_{o=m_{1} k}^{m_{1}(i-1)+m_{1}-1} \sum_{o_{1}=m_{1} k}^{o} \frac{\left(\mu_{1} U_{k}\right)^{o} 1}{o_{1} !} \frac{\left\{\mu_{1}\left(U_{i}-U_{k}\right)\right\}^{o-o_{1}}}{\left(o-o_{1}\right) !} e^{-\mu_{1} U_{i}}}{\sum_{o=m_{1} k}^{\infty} \frac{\left(\mu_{1} U_{k}\right)^{o}}{o !} e^{-\mu_{1} U_{k}}} \frac{m_{1} i-o}{\mu_{1}}(8)
\end{aligned}
$$

\section{Lemma 3.}

$$
\begin{aligned}
& \text { For } i=k+1, k+2, \cdots, n \text {, } \\
& E\left[S_{i} \mid \sum_{i=1}^{k} T_{i}>U_{k}\right] \\
& =\frac{1}{\sum_{o=0}^{m_{1} k-1} \frac{\left(\mu_{1} U_{k}\right)^{o}}{o !} e^{-\mu_{1} U_{k}}}\left(\frac{m_{2}}{\mu_{2}} \sum_{o=0}^{m_{1} k-1} \frac{\left(\mu_{1} U_{i}\right)^{o}}{o !} e^{-\mu_{1} U_{i}}\right. \\
& +\frac{m_{2}}{\mu_{2}} \sum_{o=m_{1} k}^{m_{2}(i-2)+m_{2}-1-m_{2} k+m_{1} k} \int_{U_{k}}^{U_{i}} \frac{\left(\mu_{1} x\right)^{m_{1}(k-1)+m_{1}-1}}{\left\{m_{1}(k-1)+m_{1}-1\right\} !} e^{-\mu_{1} x} \\
& \frac{\left\{\mu_{2}\left(U_{i}-x\right)\right\}^{o-m_{2} k}}{\left(o-m_{2} k\right) !} e^{-\mu_{2}\left(U_{i}-x\right)} \mu_{1} d x \\
& +\sum_{o=m_{2}(i-1)-m_{2} k+m_{1} k}^{m_{2}(i-1)+m_{2}-1-m_{2} k+m_{1} k} \int_{U_{k}}^{U_{i}} \frac{\left(\mu_{1} x\right)^{m_{1}(k-1)+m_{1}-1}}{\left\{m_{1}(k-1)+m_{1}-1\right\} !} \\
& \left.e^{-\mu_{1} x} \frac{\left\{\mu_{2}\left(U_{i}-x\right)\right\}^{o-m_{2} k}}{\left(o-m_{2} k\right) !} e^{-\mu_{2}\left(U_{i}-x\right)} \mu_{1} d x \frac{m_{2} i-m_{2} k+m_{1} k-o}{\mu_{2}}\right)(9)
\end{aligned}
$$

\subsection{Experimental Consideration}

In this section, in order to consider the optimal switching time to minimize the total expected cost by numerical experiments of a production line case, we show a case in Fig. 2, where $T=$ $5, n=10, C_{s}^{(1)}=1, C_{p}^{(1)}=2, C_{s}^{(2)}=3, C_{p}^{(2)}=6, m_{1}=m_{3}=3$ and $C_{p}=200$.

Fig. 2 shows the behavior of the optimal switching time by change of the phase number and usual processing rate. From Fig. 2, it can be noted that when $m=1$, usual processing rates are $0.1,0.2$, and 0.3 , the optimal switching times are $2 T, 3 T$, and $6 T$; when $m=3$, usual processing rates are $0.1,0.2$ and 0.3 , the optimal switching times are $3 T, 4 T$ and $7 T$, respectively.

From Fig. 2, it also can be noted that the rate should be switched to a faster one ahead of time when the usual processing speed is slow. This is because the delay cost could be decreased.

\section{Conclusion}

Due to the customer needs of cost ruduction and leadtime shorting, agile change of the production plan becames an important issue. In any social system or production process, the result and efficiency of a certain production cycle period and a certain process are influenced not only by the risks that exist in the current period but also by the risks that existed in the foregoing periods. Behind above background, in this paper, in order to consider the flexible switch strategy, we considered the optimal switching point to minimize the total expected cost in limited-cycle problems with multiple periods. First, the optimal switching problem is discussed by subdividing the process with phases. Next, the mathematic formulation of the total expectation is presented. Finally, the policy of optimal switching strategy is investigated by Erlang case, and by a numerical experiment, the some optimal switching point also have been shown, too. 


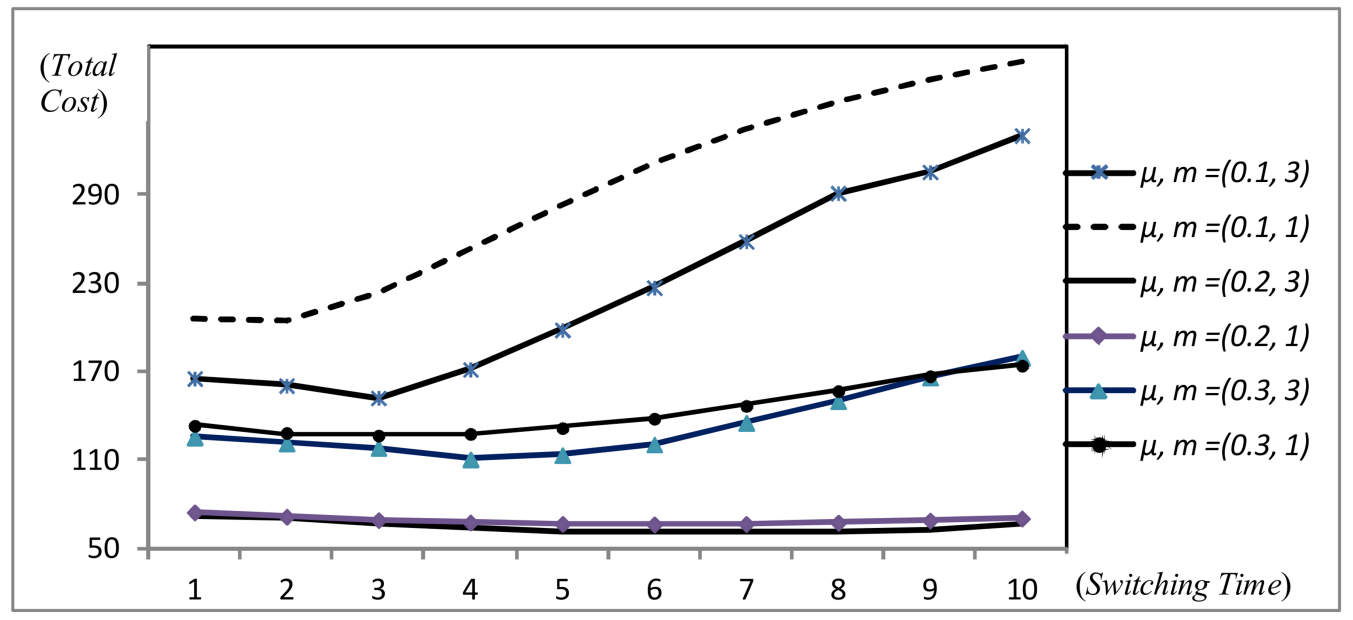

Fig. 2 Behavior of the Optimal Switching Time by Change of the Phase Number and Usual Processing Rate

\section{Acknowledgments}

This research was partially supported by the Ministry of Education, Science, Sports and Culture, a Grant-in-Aid for Scientific Research(C), 23510161.

\section{References}

[1] Eswaramoorthia, M., Kathiresan, G.R., Jayasudhan, T.J., Prasad, P.S.S., and Mohanram, P.V., 2012. Flow index based line balancing: a tool to improve the leanness of assembly line design. International Journal of Production Research. 50(12), pp. 3345-3358.

[2] Chen, Y., Lu, Y., and Xu, M., 2012. Optimal Inventory Control Policy with Inventory-Level-Dependent Demand, Naval Research Logistics, 59(6), pp. 430-440.

[3] Mejia, G., Montoya, C., Cardona, J., and Castro, A.L., 2012. Petri nets and genetic algorithms for complex manufacturing systems scheduling, International Journal of Production Research, 50(3), pp. 791-803.

[4] Yamamoto, H., Sun, J., and Matsui, M., 2010b. A Study on Limited-Cycle Scheduling Problem with Multiple Periods. Computer \& Industrial Engineering, 59(4), pp. 675-681.

[5] Yamamoto H., Matsui M. and Liu J., 2006. A basic study on a limited-cycle problem with multi periods and the optimal assignment problem. Journal of Japan Industrial Management Association 57(1), pp. 23-31 (in Japanese).

[6] Matsui, M., 2008. Manufacturing and Service Enterprise with Risk: A Stochastic Management Approach. Springer.

[7] Verzijl, J. J., 1976. Production Planning and Information Systems. Macmillan, London, 16-17.

[8] Enns, S.T., 2001. MRP performance effects due to lot size and planned lead time settings, International Journal of Production Research, 39 (3), pp. 461-480.

[9] Benders, J., 2002. The origin of period batch control (PBC). International Journal of Production Research. 40(1), pp. 1-6.

[10] Xia, W.J., and Wu, Z.M., 2005. Hybrid particle swarm optimization approach for multiobjective flexible job-shop scheduling problems, Control and Decision, 20(2), pp. 137141.

[11] Wu, X., and Zhou, X., 2008. Stochastic scheduling to minimize the expected maximum lateness, European Journal of Operational Research, 190(1), pp. 103-115.

[12] Safaei, N., and Tavakkoli-Moghaddam, R., 2009. Integrated Multi-Period Cell Formation and Subcontracting Production Planning in Dynamic Cellular manufacturing Systems, Interna- tional Journal of Production Economics, 120 (2), pp. 301-314.

[13] Porkka, P., Vepsalainen, A.P.J., and Kuula, M., 2003. Multiperiod Production Planning Carrying Over Set-up Time, International Journal of Production Research, 41 (6), pp. 11331148.

[14] El Hafsi, M., and Bai, S.X., 1998. Multi-period Production Planning with Demand and Cost Fluctuations, Journal of Mathematical and Computer Modelling, 28 (3), pp. 103-119.

[15] Li, Y., Zhang, J., Chen, J., and Cai, X., 2010. Optimal Solution Structure for Multi-period Production Planning with Returned Products Remanufacturing, Asia-Pacific Journal of Operational Research, 27 (5), pp. 629-648.

[16] Wright, Q.W., 1974. Production and Inventory Management in the Computer Age. Canners Publishing Co..

[17] Bergamashi, D., Cigolin, R., Perona, M., and Portiol, A., 1997. Order review and release strategies in a job shop environment: a review and a classification. International Journal of Production Research. 35(2), pp. 399-420.

[18] Swamidass, P. M., 2000. Encyclopedia of Production and Manufacturing Management. Kluwer, Boston.

[19] Yamamoto H., Matsui M., and Bai X.S., 2007. A Branch and Bound Method for the Optimal Assignment during a Limitcycle Problem with Multiple Periods. Journal of Japan Industrial Management Association 58(1), pp. 37-43 (in Japanese).

[20] Sun, J., Yamamoto, H., and Matsui, M., 2010. A study of optimal switching problem in limited-cycle with multiple periods, Journal of Zhejiang University-SCIENCE A (Applied Physics \& Engineering), 11(12), pp. 933-937.

[21] Yamamoto, H., Sun, J., Oishi, T., and Matsui, M., 2010a. A Study of the Optimal Switching Point of the Limited-Cycle Problem with Multiple Periods. Journal of Japan Industrial Management Association, 61(4), pp. 234-243 (in Japanese)

[22] Kong, X., Sun, J., Yamamoto, H., and Matsui, M., 2010, A Study of an Optimal Arrangement of a Processing System with twoKinds of Workers in a Limited-Cycle Problem with Multiple Periods, Proceedings of Asia Pacific Industrial Engineering \& Management Systems Conference, (on CD-ROM).

[23] Week, J.K., 1979. Optimizing planned lead times and delivery dates. 21st Annual Conference Proceedings, APICS, pp. 177188.

[24] Matsui, M., 2005. A Management cycle model: switching control under lot processing and time span. Journal of Japan Industrial Management Association 56(4), pp. 256-264.

[25] Matsui, M., Shingu, T. and Makabe, H., 1977. Conveyor- 
serviced production system: an analytic framework for stationcentered approach by some queueing formulas. Preliminary Report of the Operations Research Society of Japan, pp. 104107 (in Japanese).

Jing SuN

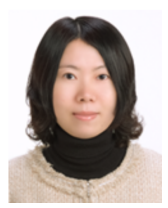

is an assistant professor in Nagoya Institute of Technology, also a guest investigator of Aoyama Gakuin University, Japan. She received her Dr. Eng. degree from The University of Electro-Communications, Japan. Her current research interests are operations research, statistical quality management, and ERP/SCM. She is now the chairman of the international affair committee of Japan Industrial Management Association (JIMA), a member of board of directors of Asian Association of Management and Applications.

\section{Xianda Kong}

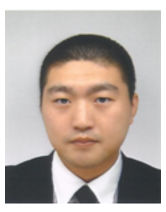

is a doctoral candidate of Tokyo Metropolitan University, Japan. He is a member of Japan Industrial Management Association (JIMA), a member of International Association of Engineers (IAENG), and a member of the Institute for Operations Research and the Management Sciences (INFORMS). His research interests include optimal worker allocation in a production system, manage-

ment engineering.

\section{Hisashi Yамамото}

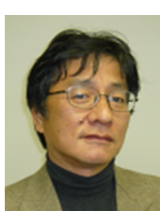

is a Professor in Faculty of System Design, Tokyo Metropolitan University, Japan. He received the B.S., M.S. and Doctoral degree (Dr. Eng.) in industrial engineering from Tokyo Institute of Technology, Japan. His main research interests are optimizations based on the reliability engineering. He worked as the Chair of IEEE Reliability society Japan Chapter, the Editor-in-chief of JIMA and REAJ. He is now the Chair of APARM2012 Program committee and a member of IEICE EA committee.

\section{Masayuki Matsui}

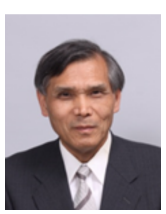

is a Professor in the Kanagawa University, and also the former President of Japan Industrial Management Association (JIMA), Japan. He received a Deng in research on conveyor-serviced production systems from Tokyo Institute of Technology, Japan. He was a Visiting Scholar of UC Berkeley and Purdue University in 1996 and 1997. His recent research interests are industrial engineering production and operations management, management theory and ERP/SCM; operations research, quality management and artificial intelligence. He was the Editor (2000-2003) and Director (2003-2005) of the JIMA journal and is a senior member of IIE. 